\title{
INEQUALITIES FOR QUERMASSINTEGRALS OF (NEW) $p$-PARALLEL BODIES
}

\section{YINGYING LOU* AND ZHENBING ZENG}

Abstract. In this paper, we define a new family of convex bodies related to the family of $p$ parallel bodies, which is determined by the 0 -extreme normal vectors, and establish some inequalities for their quermassintegrals.

Mathematics subject classification (2020): 52A20, 52A39, 52A40.

Keywords and phrases: $p$-parallel body, mixed volume, 0 -extreme normal vector.

\section{REFERENCES}

[1] W. J. FIREY, p-means of convex bodies, Math. Scand. 10, (1962), 17-24.

[2] R. J. Gardner, D. Hug AND W. Weil, Operations between sets in geometry, J. Eur. Math. Soc., 15, 6 (2013), 2297-2352.

[3] G. H. Hardy, J. E. Littlewood and G. Pólya, Inequalities, Cambridge, at the University Press, 1952, 2 d ed.

[4] M. A. HernÁndez Cifre And E. SaORín, On differentiability of quermassintegrals, Forum Math., 22, 1 (2010), 115-126.

[5] Y. Lou, D. XI AND Z. Zeng, Further inequalities and properties of p-inner parallel bodies, Canad. J. Math., http://dx.doi.org/10.4153/S0008414X20000796.

[6] E. LutwaK, D. YANG AND G. Zhang, The Brunn-Minkowski-Firey inequality for nonconvex sets, Adv. Math., 48, (2012), 407-413.

[7] A. R. MARTínEZ FERnÁNDEZ, Going further in the $L_{p}$-Brunn-Minkowski theory: a p-difference of convex bodies, $\mathrm{Ph}$. D. Thesis, University of Murcia (2016).

[8] A. R. Martínez FernándeZ, E. SaORÍn GómeZ AND J. YePes NiCOLÁs, p-difference: a counterpart of Minkowski difference in the framework of the $L_{p}$-Brunn-Minkowski theory, Rev. R. Acad. Cienc. Exactas Fís. Nat. Ser. A Mat. RACSAM, 110, 2 (2016), 613-631.

[9] J. R. SAngwine-YAGER, Inner Parallel Bodies and Geometric Inequalities, Ph. D. Thesis, University of California Davis (1978).

[10] R. SCHNEIDER, On the Aleksandrov-Fenchel inequality, In Discrete Geometry and Convexity (eds. J. E. Goodman, E. Lutwak, J. Malkevitch and R. Pollack), Ann. New York Acad. Sci., 440, 1 (1985), $132-141$.

[11] R. SchneIder, Convex bodies: The Brunn-Minkowski Theory, second expanded ed., Encyclopedia of Mathematics and Its Applications, 151, Cambridge University Press, Cambridge, 2014. 